\title{
Nutzung der Chancen der Digitalisierung durch digitale Fähigkeiten - Unsicherheit bei langfristigen Veränderungen bewältigen
}

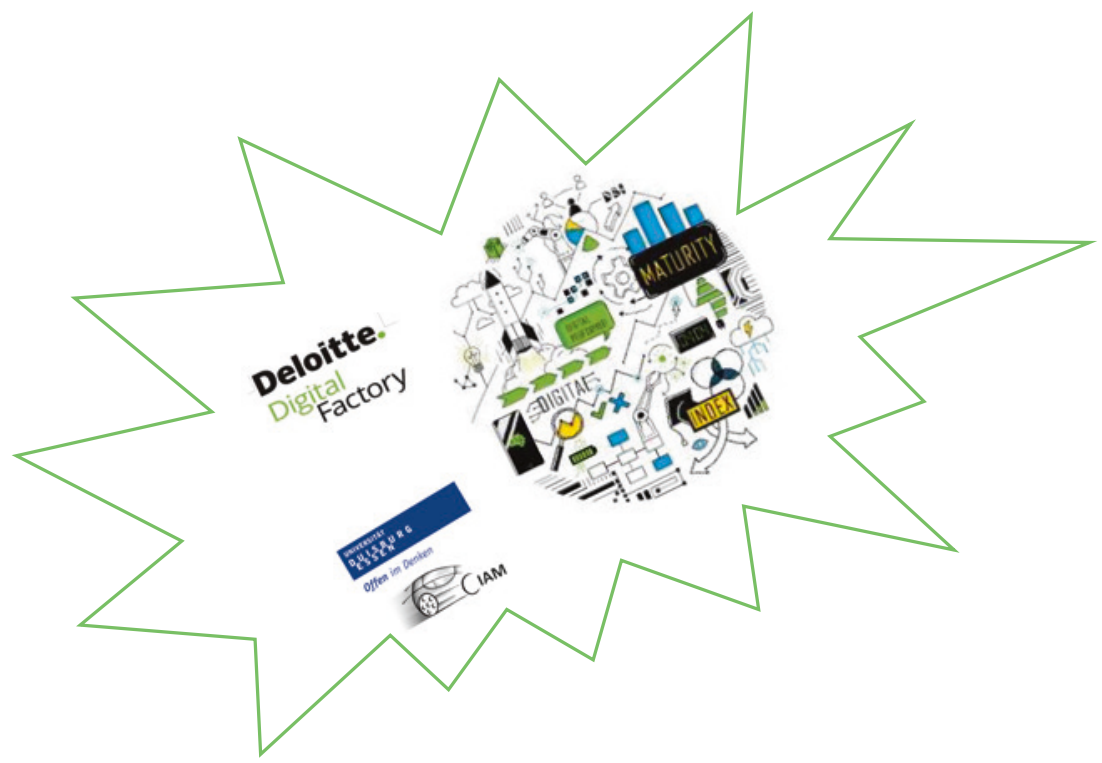

Elektronisches Zusatzmaterial Die elektronische Version dieses Kapitels enthält Zusatzmaterial, das berechtigten Benutzern zur Verfügung steht. https://doi. org/10.1007/978-3-658-31456-9_4. Die Videos lassen sich mit Hilfe der SN More Media App abspielen, wenn Sie die gekennzeichneten Abbildungen mit der App scannen. 
Wir wollen in diesem Kapitel zeigen, dass der Umgang mit der Digitalisierung Veränderungsfähigkeiten (Dynamic Capabilities) erfordert.

1. Diese Fähigkeiten helfen dabei, durch Verbesserung und Veränderung von Prozessen, Angeboten und Geschäftsmodellen (Kap. 3) die Chancen der Digitalisierung (Kap. 2) zu nutzen, weil sie es ermöglichen, die hohe Unsicherheit und Komplexität durch die Digitalisierung als langfristige Veränderung zu bewältigen.

2. Veränderungsfähigkeiten erlauben

(1) die Wahrnehmung und das Erkennen von Umfeldveränderungen,

(2) das Ergreifen neuer strategischer Optionen und

(3) die Neu-Zusammenstellung von operativen Fähigkeiten (Operational Capabilities).

Veränderungsfähigkeiten - wie auch operative Fähigkeiten - sind für Unternehmen entscheidend, um im digitalen Wandel nicht zu spät zu kommen.

Die Digitalisierung erfordert nicht nur eine Neuausrichtung der unternehmerischen Leistung (Kap. 3), sondern auch der digitalen Fähigkeiten und treibt auch darüber die Transformation von Unternehmen.

In Zeiten langfristiger diskontinuierlicher Veränderungen im Zuge der Digitalisierung brauchen Unternehmen die Fähigkeit, Umfeldveränderungen wahrzunehmen und zu erkennen (Sensing), ggf. neue strategische Optionen (Verbesserung oder Veränderung der Prozesse, Angebote und Geschäftsmodelle, vgl. Kap. 3) zu ergreifen (Seizing) und die operativen Fähigkeiten, d. h. die Kompetenzbasis, entsprechend anzupassen (Reconfiguring). Diese Veränderungsfähigkeiten treiben damit die Veränderung der operativen Fähigkeiten bzw. Kompetenzen sowie die Digitalisierung von Prozessen, Angeboten und Geschäftsmodellen und stützen die Auswirkungen auf die Gewinne (Abb. 4.1).

Das Zusammenspiel der unterschiedlichen Veränderungsfähigkeiten kann bereits bei kontinuierlichen Umfeldveränderungen dauerhafte Wettbewerbsvorteile schaffen $^{1}$ - egal ob die Veränderungen schwach und selten auftreten in einem weitgehend stabilen Umfeld wie etwa der Zementindustrie oder stark und häufig im dynamischen Umfeld wie der Computer- oder Elektronikbranche. Verändert sich die Kompetenzbasis, werden zumindest einfache Veränderungsfähigkeiten (,first order dynamic capabilities“2) gebraucht. 


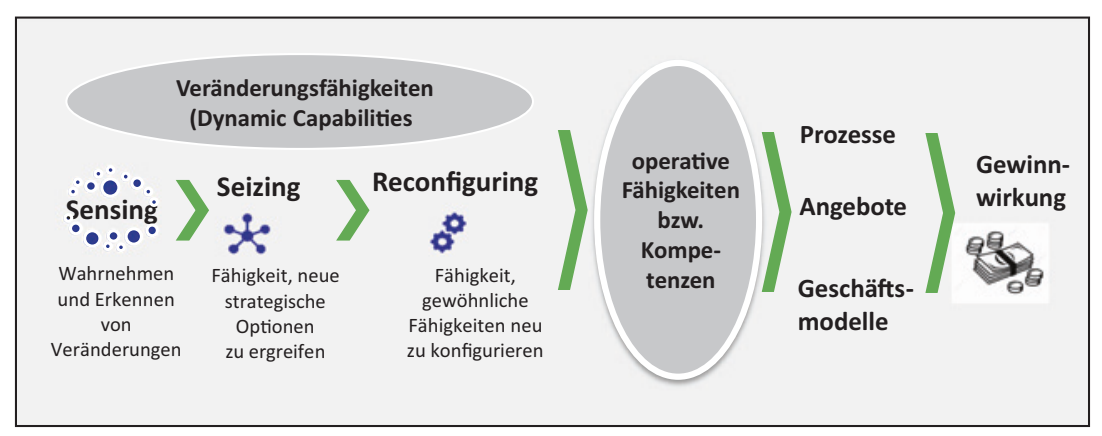

Abb.4.1 Veränderungsfähigkeiten und ihre Gewinnwirkung. (Quelle: Proff 2019 und Knobbe, 2020 bezogen z. B. auf Teece 2007)

Wichtiger werden Veränderungsfähigkeiten bei diskontinuierlichen Veränderungen im Umfeld, wie sie die Digitalisierung auslöst. ${ }^{3}$ Da hierbei die operativen Fähigkeiten nicht mehr einfach nur angepasst werden können, werden sehr weitreichende Veränderungsfähigkeiten nötig, die es erlauben, die bereits vorhandenen Veränderungsfähigkeiten ihrerseits zu verändern (,second order dynamic capabilities“4). Solche Umfeldveränderungen sind stark, kommen unerwartet und treten in einem vormals weitgehend stabilen Marktumfeld wie z. B. dem der Automobil- oder Chemieindustrie in der Regel innerhalb eines längeren Zeitraums oft nur einmal auf. Deshalb fehlt den Unternehmen dieser Branchen die Erfahrung im Umgang mit ihnen. Eine einfache Anpassung der operativen Fähigkeiten durch Weiterentwicklung im Zeitablauf durch ,first order dynamic capabilities“ reicht deshalb nicht aus. Stattdessen sind weitreichendere ,second order dynamic capabilities“ erforderlich, um die vorhandenen Veränderungsfähigkeiten zu verändern. ${ }^{5}$

Daraus folgt eine zweite Grundhypothese in Ergänzung zu Abb. 3.1: Die Gewinnwirkung der Digitalisierung ist umso höher, je stärker die Veränderungsfähigkeiten aktiviert werden, die die operativen Fähigkeiten neu konfigurieren (vgl. Abb. 4.2). 


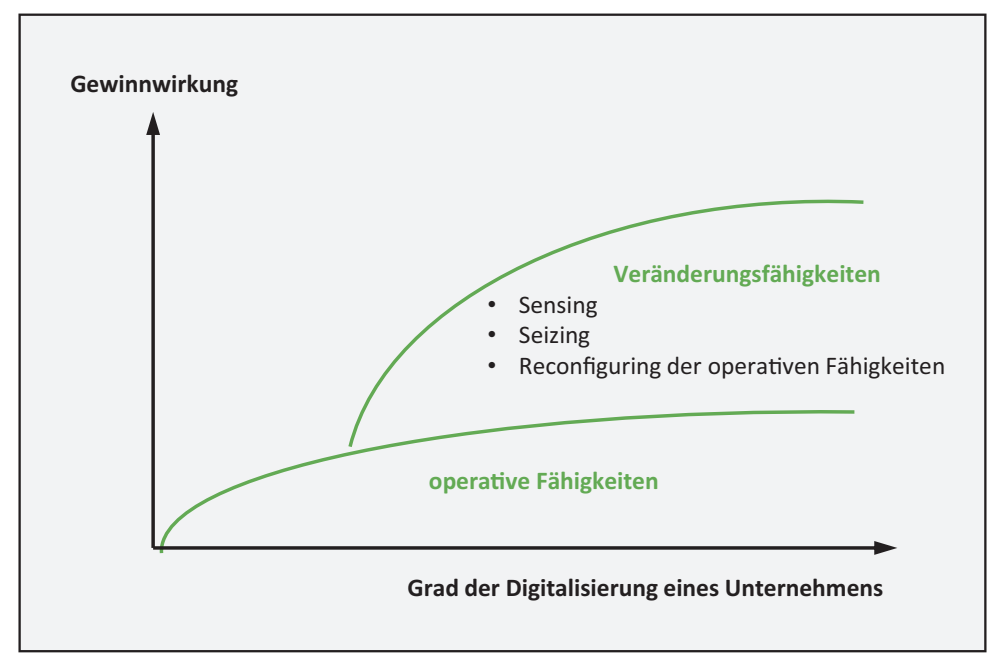

Abb.4.2 Zweite Grundhypothese zur Gewinnwirkung digitaler Fähigkeiten. (Quelle: eigener Entwurf, begründet in Kap. 2)

Am Beispiel Netflix (vgl. den nachfolgenden Kasten) lässt sich zeigen, wie wichtig die Veränderungsfähigkeiten Sensing, Seizing und Reconfiguring bei einer (weitreichenden) Digitalisierung sind: Wahrnehmung von Umfeldveränderungen, schnelle Entscheidungsfindung und Rekonfiguration der operativen Fähigkeiten ermöglichten es Netflix, zunächst auf die Weiterentwicklung des World Wide Web mit Onlinevertrieb (eCommerce) zu reagieren sowie später die steigenden Internetgeschwindigkeiten für die Einführung von StreamingDiensten über eine Streaming-Plattform zu nutzen, d. h. für Angebote, Filme und Serien online zu schauen. Die zunehmende Entkopplung von Systemen und Netzwerken macht heute auch eine Integration in neue Hardware möglich, d. h. die Zusammenarbeit mit Partnern, die Netflix in ihr System integrieren (NetflixButton). Zudem können eigene Filme entsprechend den Wünschen der Kunden, die die Netflix-Plattform nutzen, gedreht werden (vgl. folgende Grafik): 


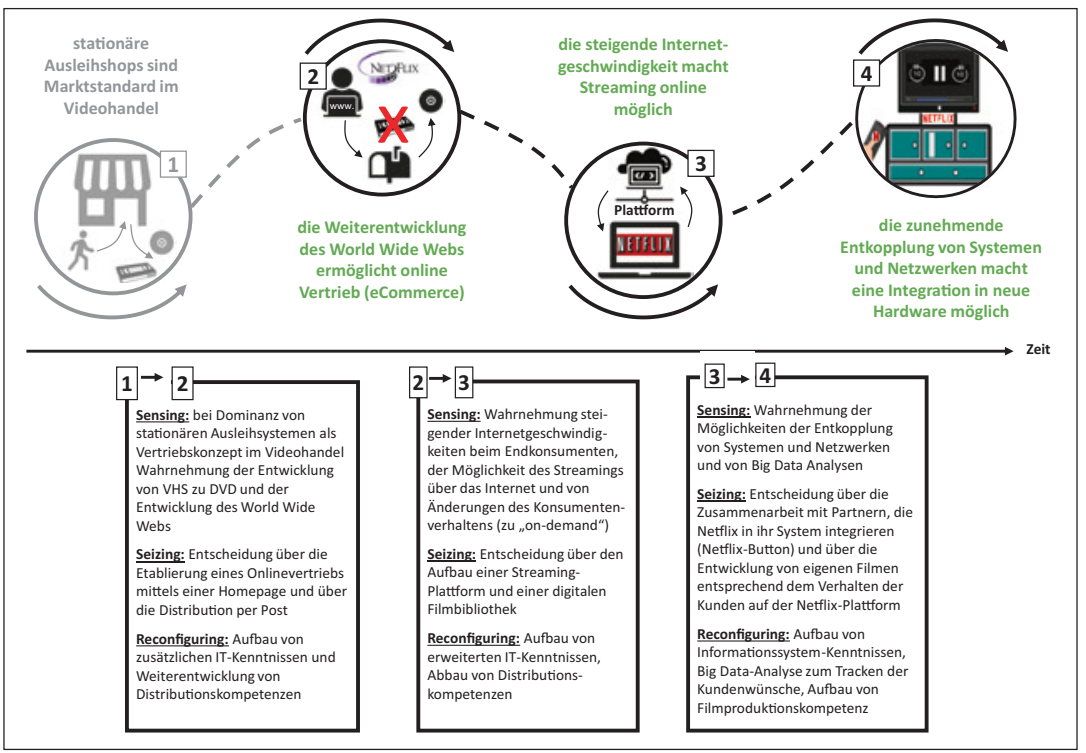

Im Folgenden soll nun gezeigt werden, dass Veränderungsfähigkeiten helfen, die Unsicherheit zu bewältigen (Abschn. 4.1). Anschließend werden die Veränderungsfähigkeiten des Sensing, Seizing und Reconfiguring genauer betrachtet (Abschn. 4.2) sowie die operativen Fähigkeiten, die sie verändern, dargestellt (Abschn. 4.3).

\subsection{Bewältigung von Unsicherheit durch Veränderungsfähigkeiten}

Veränderungsfähigkeiten ermöglichen die (Weiter-)Entwicklung von operativen Fähigkeiten bzw. Kompetenzen im Zeitablauf. ${ }^{6}$ Sie werden wichtig, wenn der Aufbau von Kompetenzen z. B. bei veränderter unternehmerischer Ressourcenbasis oder ungewollter Diffusion von Wissen aus dem Unternehmen gestört wird. ${ }^{7}$ Sie werden aber auch bedeutsam, wenn sich z. B. durch die Digitalisierung das Umfeld stärker und häufiger als zuvor und v. a. diskontinuierlich verändert und die Wahrnehmung der Nutzenstiftung der eigenen Angebote durch die Kunden verändert. ${ }^{8}$ Das liegt an der hohen Unsicherheit bezüglich der Entwicklung der Digitalisierung und der Chancen, die sie bietet. Denn zumindest kurz- und mittelfristig birgt sie das Risiko deutlich höherer Kosten durch die zunächst steigende Komplexität. ${ }^{9}$ 
Es stellt sich die Frage, wieso Komplexität und damit Unsicherheit bei einer Technologie anwachsen, die es doch ermöglichen soll, Komplexität zu verringern. Dafür gibt es drei Gründe:

- Zum einen werden in einer Übergangsphase die traditionellen Arbeitsprozesse, Angebote und Geschäftsmodelle zunächst noch weiterentwickelt. So können z. B. bei Substitution starrer Linien durch flexible Werkzeugmaschinen Produktionsprozesse weiter optimiert werden. Dies ist ein wichtiges Instrument der Industrie 4.0, ${ }^{10}$ um Kosten dieser Prozesse zu reduzieren und die Fähigkeit zu verbessern, Produktvarianten zu bearbeiten. Für das Management nimmt die Komplexität zunächst jedoch zu.

- Daneben ist in der schnellen und agilen Digitalwirtschaft der gründliche ingenieursgetriebene Ansatz vieler Unternehmen in kapitalintensiven Branchen wie der Chemie- und Automobilindustrie eher nachteilig. ${ }^{11}$ Deshalb müssen die teilweise sehr verschiedenen Lebenszyklen z. B. von Fahrzeugen und Konsumgüterelektronik überbrückt und synchronisiert werden. ${ }^{12}$ Außerdem gilt es, die Anschlussfähigkeit (Konnektivität) der Kundenendgeräte und der darauf verfügbaren Anwendungen sicherzustellen. Für das Management steigt auch dadurch die Komplexität sehr stark.

- Zudem verstärkt sich der Wettbewerb durch branchenfremde Konkurrenten, insbesondere durch IT-Unternehmen, die in der Lage sind, ,schnell in neue Geschäftsfelder vorzustoßen und digitale Angebote dank ihrer Marktmacht und ihrer Expertise mit Datenanalytik in kurzer Zeit zu etablieren"13 und damit andere Unternehmen von ihren Geschäftsmodellen abhängig machen. Dadurch erhöht sich nochmals die Komplexität.

Veränderungsfähigkeiten, vor allem die Wahrnehmung und das Erkennen von Umfeldveränderungen, und darauf bezogen das Ergreifen strategischer Optionen sowie die Neukonfiguration der operativen Fähigkeiten helfen, Unsicherheit und Komplexität zu bewältigen. Damit lassen sich die Chancen der Digitalisierung schneller nutzen. Veränderungsfähigkeiten bilden zudem die Basis für rechtzeitige Entscheidungen mit hoher Qualität. Voraussetzungen sind dafür gemäß der modernen Entscheidungstheorie (1) Gruppenentscheidungen und (2) die Vermeidung von Verzögerungen bei der Umsetzung der Veränderungsfähigkeiten (vgl. Abb. 4.3). 


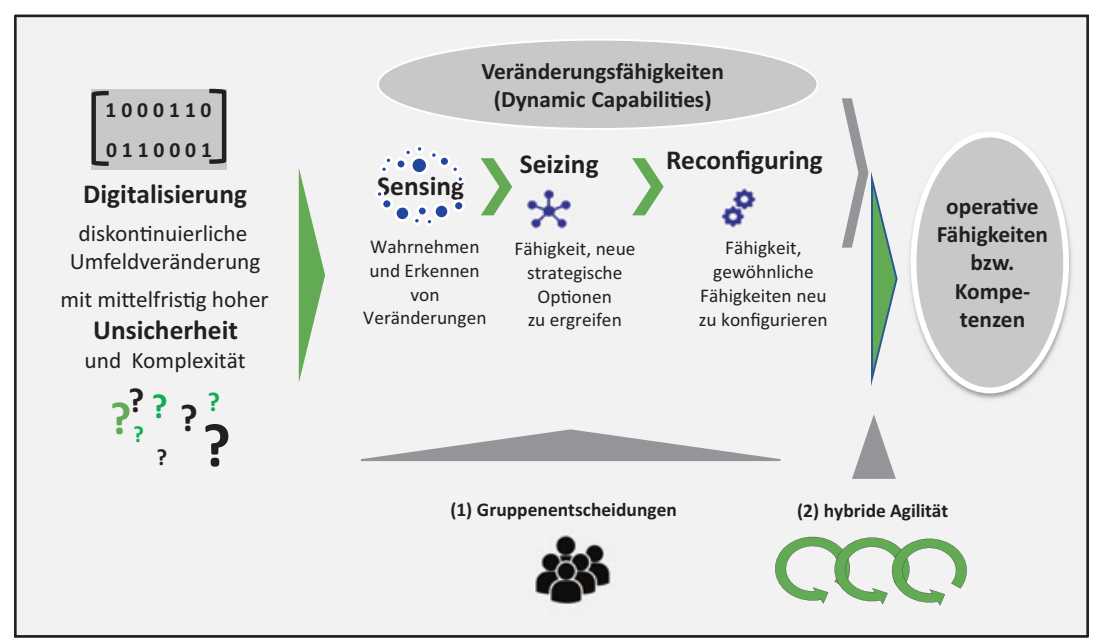

Abb. 4.3 Bewältigung von Unsicherheit durch Veränderungsfähigkeiten. (Quelle: eigener Entwurf in Erweiterung von Abb. 4.1)

$\mathrm{Zu}$ (1): Gruppenentscheidungen sind gemeinsame Entscheidungen mehrerer kompetenter Entscheidungsträger, ${ }^{14}$ die unterschiedliches Wissen einbringen und - wenn nötig - zusätzliche Informationen suchen. In einem modernen Entscheidungsmodell $^{15}$ wird die Qualität einer Gruppenentscheidung, z. B. über relevante Umfeldveränderungen und strategische sowie operative Reaktionen darauf, in Abhängigkeit von der Breite des entscheidungsrelevanten Wissensstandes der Entscheidungsträger erklärt. ${ }^{16}$ Die Varianz des Know-hows in der Gruppe zeigt das unterschiedliche Wissen der Entscheider. Haben alle Entscheider ein ähnliches Know-how, ist der Wissensstand eng, unterscheidet sich das Knowhow der wichtigsten Entscheider, deckt es eine gewisse Bandbreite ab. ${ }^{17}$

In Erweiterung dieses Entscheidungsmodells kann eine negative Beziehung angenommen werden zwischen der Qualität einer Gruppenentscheidung und der Unsicherheit, die bei komplexen Entscheidungsproblemen über das mögliche Ergebnis der Entscheidung besteht. In einer komplexen und unsicheren Entscheidungssituation ist die Entscheidungsqualität oft sehr schlecht. Selbst wenn die Entscheidungsträger bei hoher Unsicherheit einen gewissen Rückgang der Entscheidungsqualität akzeptieren müssen, ${ }^{18}$ sollte die Entscheidung auf eine 
Gruppe von kompetenten Entscheidungsträgern delegiert werden, die Wissen für das Managementteam aufbereitet und das entscheidungsrelevante Wissen erhöht. ${ }^{19}$ Alternativ bleibt nur abzuwarten, bis das entsprechende Wissen und damit die Entscheidungsqualität deutlich zunehmen. Das kann aber lange dauern - ungenutzte Zeit, in der die Gefahr besteht, keine oder falsche Entscheidungen zu treffen.

$\mathrm{Zu}$ (2): Bei der Umsetzung von Veränderungsfähigkeiten kann es zu Verzögerungen kommen, ${ }^{20}$ die auf Veränderungsbarrieren hindeuten. Verzögerungen bei der Suche nach relevanten Umfeldveränderungen und nach möglichen Reaktionen können sich z. B. ergeben, wenn das Top-Management das Sensing und Seizing nicht (richtig) unterstützt. Weitere Verzögerungen sind bei der Neukonfiguration der operativen Fähigkeiten bzw. der Kompetenzbasis der Unternehmen zu erwarten, z. B. wenn gemeinsame Werte in einer innovativen Unternehmenskultur fehlen. So hat z. B. die Untersuchung von Veränderungsfähigkeiten in der Automobilindustrie von Proff und Knobbe (2020, vgl. auch Kap. 1) gezeigt, dass 89 der befragten 96 Automobilzulieferer zwar die großen, diskontinuierlichen Veränderungen durch die Digitalisierung wahrnehmen, die meisten dieser Unternehmen aber deren Bedeutung unterschätzen. Deshalb wird gerade in der Automobilindustrie die Implementierung digitalisierter und vor allem digitaler Prozesse, Angebote und Geschäftsmodelle (vgl. Kap. 3) erst langsam vorangetrieben ${ }^{21}$ („Revolutions always start silently“, KPMG 2019, S. 7) und es zeigen sich Widerstände gegen die Digitalisierung. Dadurch wird wertvolle Anpassungszeit vertan, die gegenüber neuen branchenfremden Wettbewerbern bei Mobilitätsdiensten wie Google und Waymo ${ }^{22}$ fehlen wird. ${ }^{23}$

Im Umgang mit Komplexität und Unsicherheit gerade in der digitalen Transformation ist es deshalb wichtig, nicht nur Veränderungsfähigkeiten zu aktivieren, sondern sie auch umzusetzen. Dabei helfen neben der Unterstützung durch das Top-Management und einer innovativen Unternehmenskultur z. B. auch Transformationsfähigkeiten, vor allem Agilität. ${ }^{24}$ Damit ist allerdings nicht jede Form der strategischen Flexibilität gemeint, ${ }^{25}$ sondern organisationale Agilität ${ }^{26}$ als Fähigkeit der Umsetzung von Veränderungsfähigkeiten in der Organisation. Bei Veränderungen der Rahmenbedingungen ermöglicht diese eine effektive und effiziente Neu-Konfiguration von Ressourcen auf wertschaffende und -sichernde Aktivitäten mit hohen Ertragspotenzialen. Sie ermöglicht kurze verbindliche Entscheidungsprozesse, die ausgehend von minimal lebensfähigen 
Produkten den optimalen Weg bestimmen und dabei stabile operative Fähigkeiten aufbrechen, die oft starr in statischen Routinen ablaufen. ${ }^{27}$ Es ist dabei allerdings zu berücksichtigen, dass trotz des Trends zu weniger Kapitaleinsatz (,,asset light"-Strategien ${ }^{28}$ ) gerade in kapitalintensiven Branchen wie der Automobil- und Chemieindustrie bei tiefgreifenden technologischen Veränderungen durch die Digitalisierung, die mit hoher Unsicherheit verbunden sind, Agilität nicht unbegrenzt möglich ist. Angesichts der hohen Fixkosten müssen in solchen kapitalintensiven Branchen stattdessen Größen- und Verbundvorteile auch im Übergang zu neuen digitalen Technologien optimiert werden. ${ }^{29}$ Damit ist es nötig, Agilität unter der Nebenbedingung der Optimierung von Größen- und Verbundvorteilen zu erreichen, was als hybride Agilität bezeichnet wird. ${ }^{30}$

\subsection{Veränderungsfähigkeiten des Sensing, Seizing und Reconfiguring}

Wie erwähnt, werden drei Veränderungsfähigkeiten unterschieden (vgl. Abb. 4.1): (1) Sensing, (2) Seizing und (3) Reconfiguring, die die operativen Fähigkeiten bzw. Kompetenzen verändern.

\section{(1) Sensing als Fähigkeit, Umfeldveränderungen wahrzunehmen}

Bei diskontinuierlichen Veränderungen mit hoher Komplexität und Unsicherheit durch die Digitalisierung ist zunächst die Fähigkeit erforderlich, Umfeldveränderungen wahrzunehmen und anschließend eine ,kollektive Sinngebung “31 zu beginnen (,sensing and shaping of opportunities") ${ }^{32}$. Dazu sind diese Veränderungen vor dem Hintergrund des unternehmerischen Zielsystems zu interpretieren und strategische Ziele sowie Instrumente festzulegen, um strategische Möglichkeiten zu identifizieren.

Unternehmen brauchen dafür Analysesysteme und individuelle Fähigkeiten des Lernens und Erkennens, Filterns, Formens und Kalibrierens von Chancen. Damit können sie Innovationen erschließen, die durch unternehmensinterne FuE, durch Lieferanten, in Wissenschaft und Technik sowie durch Kunden entstehen (vgl. Abb. 4.4), indem sie Prozesse des Wissensaustauschs, der Vernetzung von Praxis und Wissenschaft sowie Innovationswettbewerbe nutzen. Es geht um eine breit angelegte Suche nach Chancen, die auch aktuelle und potenzielle Kooperationspartner miteinbezieht. ${ }^{33}$ 


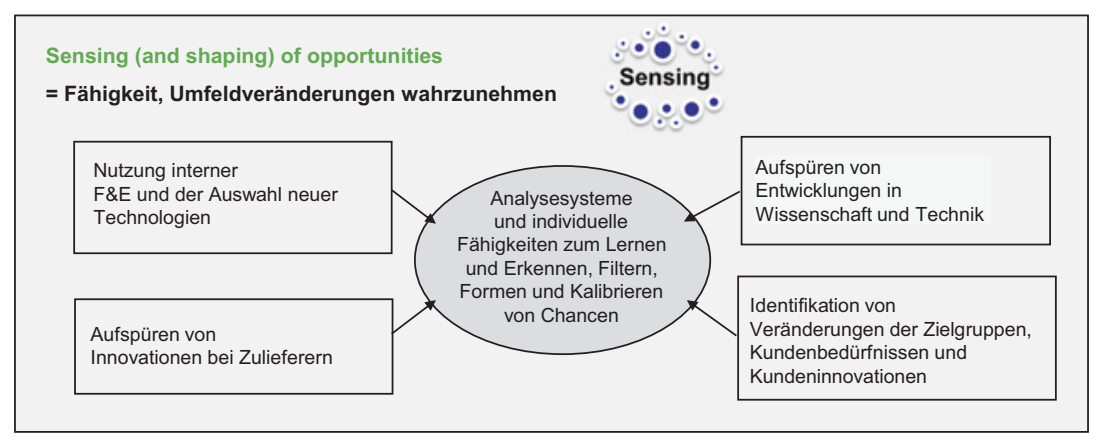

Abb. 4.4 Sensing als Wahrnehmung von Umfeldveränderungen. (vgl. Teece 2007, S. 1326)

\section{(2) Seizing als Fähigkeit, neue strategische Möglichkeiten zu ergreifen}

Bei diskontinuierlichen Veränderungen mit hoher Komplexität und Unsicherheit durch die Digitalisierung ist zudem die Fähigkeit erforderlich, die im Sensing erkannten neuen strategischen Möglichkeiten und Chancen auch zu ergreifen (Seizing of opportunities). Dafür sind geeignete Unternehmensstrukturen und -prozesse sowie Anreize zu schaffen, die sich auf vier Mikroprozesse stützen können: ${ }^{34}$ 1) Kundenlösungen und Geschäftsmodelle skizzieren, 2) Entscheidungen finden, 3) Unternehmensgrenzen bestimmen sowie 4) Loyalität und bindende Verpflichtungen aufbauen.

Um Kundenlösungen und Geschäftsmodelle skizzieren zu können, sind geeignete Technologien und Produktarchitekturen sowie Zielkunden auszuwählen, zudem Ertragsarchitekturen und Mechanismen der Wertsicherung zu entwickeln. Um weiterhin Entscheidungswege auszuwählen, ist es wichtig, kritische Entscheidungspunkte zu erkennen und Entscheidungsfehler zu vermeiden. Zudem sind Unternehmensgrenzen zu bestimmen, um Komplementaritäten zu managen und Plattformen zu kontrollieren. Dafür gilt es, die Spezifität der Vermögenswerte zu bestimmen, erfolgskritische Ressourcen zu kontrollieren und Co-Spezialisierungsvorteile, d.h. eine mehrwertstiftende Kombination aus physischen Vermögenswerten, Humanressourcen und geistigem Eigentum eines Unternehmens, zu erkennen und zu managen. Damit werden spezifische und komplexe Fähigkeiten geschaffen. Schließlich müssen Loyalität und Commitment aufgebaut werden. Dies ist möglich, wenn Unternehmen Führungsstärke demonstrieren, effektiv kommunizieren und den Einfluss nicht-ökonomischer Faktoren, Werte und Kultur auf den Erfolg erkennen (vgl. Abb. 4.5). 


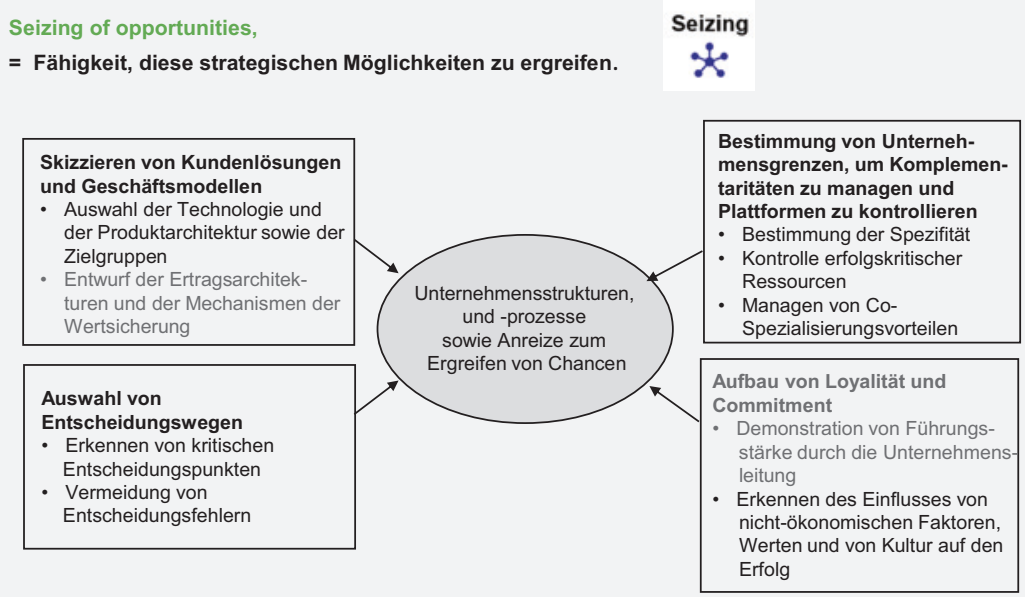

Abb.4.5 Seizing als Ergreifen neuer strategischer Möglichkeiten. (vgl. Teece 2007, S. 1326)

(3) Reconfiguring als Fähigkeit, durch Kombination und neue Zusammenstellung operativer Fähigkeiten innovative Wettbewerbsvorteile zu erzielen

Diskontinuierliche Veränderungen mit hoher Komplexität und Unsicherheit durch die Digitalisierung erfordern schließlich auch die Fähigkeit, Chancen umzusetzen, d. h. durch (Neu-)Ausrichtung der unternehmerischen Ressourcen wettbewerbsfähig zu bleiben. Dazu sind materielle und immaterielle Ressourcen zu erweitern, zu kombinieren, zu schützen, und - wenn nötig - neu zusammenzustellen (Reconfiguring), um neue, innovative Wettbewerbsvorteile zu erzielen. Dies erfordert eine ständige Anpassung und Neuausrichtung der Ressourcenbasis, wobei wiederum vier Mikroprozesse unterstützen können: ${ }^{35}$ 1) der Dezentralisierung und Modularisierung, 2) der internen Steuerung (Governance), 3) der Co-Spezialisierung und 4) des Wissensmanagements.

Um Dezentralisierung und Modularisierung $\mathrm{zu}$ erreichen und Netzwerke bilden zu können, ${ }^{36}$ müssen Unternehmen ihre Strukturen lose koppeln, Open Innovation steuern sowie Integrations- und Koordinationsfähigkeiten entwickeln. Die Organisation der internen Steuerung braucht geeignete Anreizsysteme sowie Prozesse zur Vermeidung von Opportunismus, strategischem Fehlverhalten und Gewinnverschwendung. Co-Spezialisierung erfordert eine strategische Passung der Chancen mit der Ressourcenposition und Wissensmanagement durch Lernen, Wissenstransfer, Know-how-Integration sowie Schutz von Know-how und geistigem Eigentum (Abb. 4.6). 


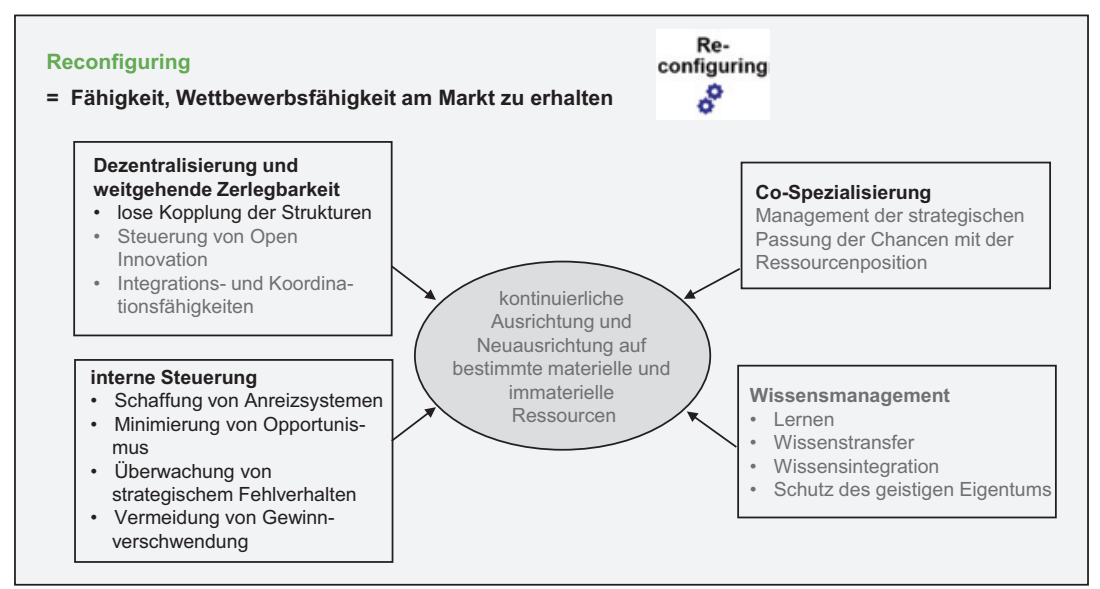

Abb. 4.6 Reconfiguring als neue Kombination von operativen Fähigkeiten. (vgl. Teece 2007, S. 1340)

Die einzelnen Veränderungsfähigkeiten (Wahrnehmen, Ergreifen und Umsetzen von Chancen) müssen umso stärker aktiviert werden, je größer und diskontinuierlicher die Veränderungen sind. Sie dürfen allerdings nicht gleichzeitig aktiviert werden, sondern müssen aufeinanderfolgen, da es sonst zu Chaos kommt und die Effektivität abnehmen wird. ${ }^{37}$

Die Veränderungen werden in der Regel durch Top-Manager (allein oder in Teams) wahrgenommen. Die erforderliche Managementfähigkeit des Sensing wird im Unternehmen durch frühere Erfahrungen und durch Lernen erworben und ist schwer imitierbar. Das Ergreifen von Möglichkeiten und die Veränderung der operativen Fähigkeiten beruhen dagegen eher auf Routinen und sind eine Aufgabe des ganzen Unternehmens. ${ }^{38}$ Dabei sind Veränderungsfähigkeiten nicht permanent aktiviert, sondern können auch deaktiviert bleiben. ${ }^{39}$ Einige Unternehmen in weitgehend stabilen Branchen haben sie aufgegeben, weil sie ungenutzt sehr hohe Kosten verursachen würden. Zudem gibt es Barrieren bei der Umsetzung von Veränderungsfähigkeiten (vgl. hierzu das nachfolgende Video 3). ${ }^{40}$ 
Video 3: Umsetzungsbarrieren in Zeiten der Digitalisierung (https://doi.org/10.1007/0000sy)

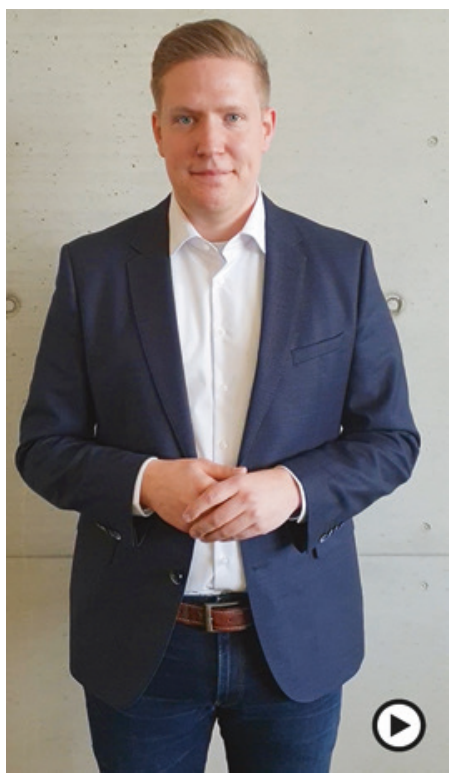

\subsection{Operative Fähigkeiten}

Gemäß dem Dynamic Capability-Ansatz müssen bei Umfeldveränderungen operative Fähigkeiten bzw. Kompetenzen verändert werden, und zwar umso schneller, je stärker die Veränderungen wahrgenommen werden und je besser die Veränderungsfähigkeiten sind. ${ }^{41}$

Bei kontinuierlichen Veränderungen stoßen solche Veränderungsfähigkeiten gemäß den Erklärungen der Kompetenzentwicklung ${ }^{42}$ eine Weiterentwicklung der operativen Fähigkeiten durch eine abwechselnde Verbesserung und Erneuerung von Kompetenzen an. ${ }^{43}$ Diese Abfolge wird in einem dynamischen Umfeld schneller und stärker erfolgen als in einem weitgehend stabilen Umfeld und muss sich auf unterschiedlich starke Lernprozesse stützen. ${ }^{44}$ Bei den diskontinuierlichen Veränderungen durch die Digitalisierung fehlen meist Kompetenzen, sodass ein Kompetenzsprung (,leapfrogging“") durch Kooperation oder Akquisition erforderlich wird. ${ }^{45}$ 
Zu den digitalen (operativen) Fähigkeiten bzw. Kompetenzen zählen

- (individuelle) digitale Fähigkeiten einzelner Mitarbeiter bzw. digitale Talente in Unternehmen und

- (organisationale) digitale Fähigkeiten wie die Unterstützung der digitalen Fähigkeiten durch das Top-Management, die Schaffung einer digitalen Unternehmenskultur und einer klaren Roadmap der digitalen Kompetenzen sowie die Erfassung der Fortschritte der digitalen Transformation in einer Scorecard. Diese Fähigkeiten zeichnen sich dadurch aus, dass sie komplex in Unternehmen verankert sind, unternehmensspezifisch sind und nicht nach außen dringen (stillgehalten werden) - damit sind sie begrenzt handel- und imitierbar. ${ }^{46}$ Stilles Wissen lässt sich nicht durch ,reverse engineering“ nachahmen. ${ }^{47}$ Die Komplexität der organisationalen Ressourcen soll sicherstellen, dass einzelne Komponenten der unternehmensspezifischen Ressourcenbasis nicht von Außenstehenden bewertet und nicht isoliert werden können. erschwert den Handel mit Ressourcen, verringert die Transparenz und erschwert damit die Nachahmung. ${ }^{48}$ Bei unternehmensspezifischen Ressourcen bestehen hohe Transaktionskosten, wenn sie aus dem Kontext gelöst werden, damit wird der Handel mit ihnen sehr teuer (vgl. hierzu auch das Video 4). ${ }^{49}$

Video 4: Operational Capabilities zwischen Wissensabfluss und Stärkung in Netzwerken (https://doi. org/10.1007/000-0sx)

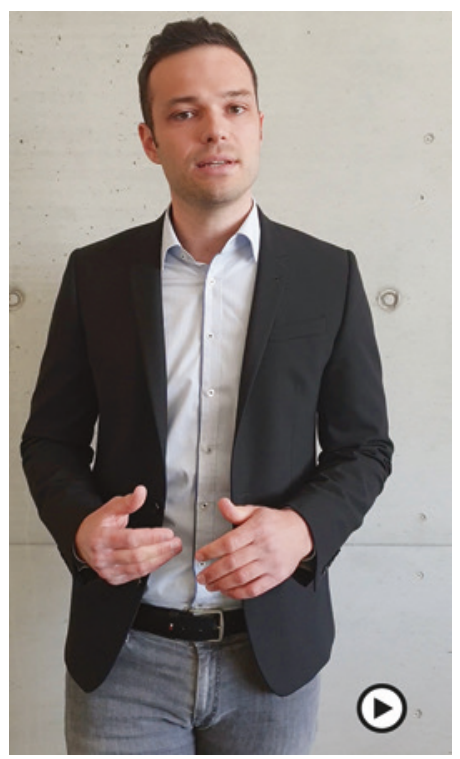


In diesem Kapitel wurde gezeigt, dass die durch die Digitalisierung veränderten digitale Fähigkeiten - Veränderungsfähigkeiten wie operative Fähigkeiten - die leistungsmäßigen Veränderungen von Prozessen, Angeboten und Geschäftsmodellen (Kap. 3) unterstützen und so helfen, die Digitalisierung zu beschleunigen.

\section{Anmerkungen zu Kapitel 4}

1. Vgl. Peteraf u. a. (2013).

2. Vgl. z. B. Schilke (2014).

3. Vgl. Knobbe, Proff (2020).

4. Vgl. Collis (1994); Zollo, Winter (2002, S. 340); Danneels (2002).

5. Vgl. auch Knobbe, Proff (2020).

6. Vgl. Teece (2014, S. 332) und darauf bezogen Proff (2019).

7. Vgl. z. B. Proff (2005).

8. Vgl. McGrath u. a. (1995) und darauf bezogen Proff (2005).

9. Vgl auch Proff (2019, Abschn. 3.3.3).

10. Vgl. Deloitte (2017).

11. Vgl. Gissler, Seibert (2015, S. 7).

12. Köhler, Wollschläger (2014, S. 72).

13. Ebd.

14. Mezias, Starbuck (2003); Talluri, van Ryzin (2004); Csaszar, Eggers (2013) und darauf bezogen Proff (2019).

15. Csaszar, Eggers (2013).

16. Vgl. Hastie, Kameda (2005).

17. Vgl. Mezias, Starbuck (2003).

18. Vgl. Proff (2019) bezogen auf Proff, Fojcik (2015).

19. Vgl. Kolb, Faure (1994, S. 128); Zartman (1994, S. 219) oder Talluri, van Ryzin (2004).

20. Vgl. Knobbe (2020).

21. Vgl. Szalavetz, 2019).

22. Vgl. Riasanow u. a. (2017).

23. Vgl. z. B. Teece, Linden (2017, S. 10).

24. Vgl. Zahra u. a. (2006): 919; Teece u. a. (2016) und darauf bezogen Proff u. a. (2020).

25. Wie z. B. bei Doz,Kosonan (2008) und Weber, Tarrba (2014).

26. Vgl. z. B. Teece u. a. (2016).

27. Vgl. ebd. (S. 25 und S. 21).

28. Vgl. Kachaner, Whybrew (2014).

29. Vgl. Proff u. a. (2020) bezogen auf Meyer, Su (2015) und Becker u. a. (2016). 
30 Vgl. z. B. Proff (2019) und Proff u. a. (2020).

31. Meier, Slembeck (1994, S. 39).

32. Teece (2007).

33. Vgl. Schreyögg, Eberl (2015).

34. Vgl. Teece (2007).

35. Vgl. ebd.

36. Schreyögg, Eberl (2015).

37. Vgl. Teece (2007, S. 1341).

38. Vgl. Zahra u. a. (2006).

39. Vgl. Ambrosini, Bowman (2009) oder Helfat, Peteraf (2009).

40. Vgl. Zollo, Winter (2002); Winter (2003).

41. Vgl. Teece (2014, S. 335 und ähnlich 2018, S. 43): „The strength of a firm's dynamic capabilities determines the speed and degree [and associated costs] to which the firm's resources can be aligned consistent with the firm's strategy [business model] and with changes in the [...] business environment".

42. Vgl. Baden-Fuller, Volberda (1997); Volberda, Baden-Fuller (1998).

43. „Cycling“, Baden-Fuller, Volberda (1997); Volberda, Baden-Fuller (1998); Proff (2005).

44. Vgl. Pentland u. a. (2012).

45. Vgl. z. B. Brezis u. a. (1991) und darauf bezogen Proff (2019).

46. Vgl. Proff (2002).

47. Vgl. Proff, 2002, S. 127 bezogen auf Nelson, Winter 1982, S. 123).

48. Vgl. ebd., bezogen u. a. Barney (1991, S. 110-111).

49. Vgl. ebd.

Open Access Dieses Kapitel wird unter der Creative Commons Namensnennung 4.0 International Lizenz (http://creativecommons.org/licenses/by/4.0/deed.de) veröffentlicht, welche die Nutzung, Vervielfältigung, Bearbeitung, Verbreitung und Wiedergabe in jeglichem Medium und Format erlaubt, sofern Sie den/die ursprünglichen Autor(en) und die Quelle ordnungsgemäß nennen, einen Link zur Creative Commons Lizenz beifügen und angeben, ob Änderungen vorgenommen wurden.

Die in diesem Kapitel enthaltenen Bilder und sonstiges Drittmaterial unterliegen ebenfalls der genannten Creative Commons Lizenz, sofern sich aus der Abbildungslegende nichts anderes ergibt. Sofern das betreffende Material nicht unter der genannten Creative Commons Lizenz steht und die betreffende Handlung nicht nach gesetzlichen Vorschriften erlaubt ist, ist für die oben aufgeführten Weiterverwendungen des Materials die Einwilligung des jeweiligen Rechteinhabers einzuholen.

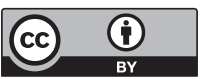

